\title{
Optimizing Noncardiac Prescription in a Cardiac Patient
}

\author{
Ashraf Kader Sahib ${ }^{1}$, Meenakshisundaram Chenniappan ${ }^{2}$
}

\begin{abstract}
Comorbidities are present in greater than $75 \%$ of cardiac patients. While choosing therapy for such patients it is important to keep in mind the complex interactions of various drugs in the background of comorbidities. Safety should be the prime concern though the efficacy of the drugs should not be compromised too much. This article touches upon the therapy of cardiac patients with anemia, diabetes mellitus, kidney disease, thyroid disorders, sleep disorders, psychiatric illness, or malignancies. Drug class, dosage, drug route, or contraindications have been highlighted for optimal management.

Keywords: Comorbidities, Drug interactions, Efficacy, Safety.

Bengal Physician Journal (2020): 10.5005/jp-journals-10070-7032
\end{abstract}

\section{INTRODUCTION}

Patients with heart disease often have multiple comorbidities that interact with the underlying cardiac condition, alter the prognosis, and may often decide the choice of therapy. Commonly encountered such conditions include anemia, chronic obstructive pulmonary disease (COPD), sleep apnea, and chronic kidney disease. This article will give an insight into the common comorbidities and heart and help in optimizing the drug therapy for those conditions to protect the heart and minimize the drug interaction with cardiac drugs.

\section{Anemia}

World Health Organization (WHO) defines anemia as $\mathrm{Hb}<13 \mathrm{~g} / \mathrm{dL}$ in men and $<12 \mathrm{~g} / \mathrm{dL}$ in women. Its prevalence in heart failure is around $20 \%$. The within-person change in hemoglobin over time is more important than the actual hemoglobin level. After adjustment for other clinical variables, in anemic patients, the short and long-term mortality of heart failure is 1.5-2-fold, patients require more frequent hospitalizations and are in worse New York Heart Association (NYHA) functional class. Guidelines recommend identifying the etiology and treating anemia appropriately. Hemoglobin follows a J/U shaped curve wherein both higher and lower levels are associated with poorer outcomes in heart failure.

Iron deficiency, B12/folate deficiencies, increased proinflammatory cytokines, reduced renal perfusion, reduced sensitivity to erythropoietin, blood loss, bone marrow disorders, hemodilution are the common causes of anemia in heart failure. A lot of importance has been attached to serum iron levels and ferritin recently because iron deficiency with or without anemia increases
${ }^{1}$ Apollo Specialty Hospitals, Trichy, Tamil Nadu, India

${ }^{2}$ The Tamil Nadu Dr MGR Medical University, Chennai, Tamil Nadu, India

Corresponding Author: Meenakshisundaram Chenniappan, The Tamil Nadu Dr MGR Medical University, Chennai, Tamil Nadu, India, Phone: +91 9944938982, e-mail: chennidr@gmail.com

How to cite this article: Sahib AK, Chenniappan M. Optimizing Noncardiac Prescription in a Cardiac Patient. Bengal Physician Journal 2020;7(3):63-69.

Source of support: Nil

Conflict of interest: None

mortality. Definition of iron deficiency in heart failure differs from other conditions of chronic inflammation and is defined as (1) ferritin $<100 \mu \mathrm{g} / \mathrm{L}$ or $(2)$ ferritin of $100-299 \mu \mathrm{g} / \mathrm{L}$ and transferrin saturation $<20 \%$. Iron deficiency rather than iron deficiency anemia should be treated in heart failure to reduce mortality.

\section{Treatment of Anemia (Table 1)}

- Identify and treat the cause of anemia. Blood transfusion should be reserved forasymptomatic patients with $\mathrm{Hb}<7 \mathrm{~g} / \mathrm{dL}$ (packed 2 cells).

- Treatment of iron deficiency.

- At present, intravenous (IV) iron is the preferred route for treatment in heart failure patients. Most studies have used IV iron sucrose (maximum dose of $200 \mathrm{mg}$ per setting) orferric carboxymaltose (maximum dose of $1000 \mathrm{mg}$ per week).

Table 1: Dosage and administration of intravenous iron

\begin{tabular}{|c|c|c|}
\hline $\begin{array}{l}\text { Intravenous } \\
\text { Iron complexes }\end{array}$ & Dosage & Administration \\
\hline Iron carboxymaltose & $\begin{array}{l}\text { IV infusion at } 15 \mathrm{mg} / \mathrm{kg}(\max \leq 1000 \mathrm{mg}) \\
\text { once/week Bolus IV injection of } 200 \mathrm{mg} / \text { day } \\
\text { ( }<3 \text { injections/week) }\end{array}$ & $\begin{array}{l}\text { IV infusion in normal saline (NS) with iron concentration }>2 \mathrm{mg} / \mathrm{mL} \\
\text { Up to } 200 \mathrm{mg} \text { : use } 50 \mathrm{~mL} \text { of NS } \\
200-500 \mathrm{mg} \text { : use } 100 \mathrm{~mL} \text { of NS, over }>6 \mathrm{~min} \\
500-1000 \mathrm{mg} \text { : use } 250 \mathrm{~mL} \mathrm{NS} \text {, over } 15 \mathrm{~min}\end{array}$ \\
\hline $\begin{array}{l}\text { Iron hydroxide } \\
\text { sucrose }\end{array}$ & $\begin{array}{l}\text { 100-300 mg/injection; } 1-3 \text { injections/week } \\
\text { with a } 48 \text {-h interval between each injection }\end{array}$ & $\begin{array}{l}\text { Slow IV infusion with a duration }>1.5 \text { hours; } 1 \text { ampoule dilated in } \\
<100 \mathrm{~mL} \text { of NS }\end{array}$ \\
\hline
\end{tabular}

(0) The Author(s). 2020 Open Access This article is distributed under the terms of the Creative Commons Attribution 4.0 International License (https:// creativecommons.org/licenses/by-nc/4.0/), which permits unrestricted use, distribution, and non-commercial reproduction in any medium, provided you give appropriate credit to the original author(s) and the source, provide a link to the Creative Commons license, and indicate if changes were made. The Creative Commons Public Domain Dedication waiver (http://creativecommons.org/publicdomain/zero/1.0/) applies to the data made available in this article, unless otherwise stated. 
- IV iron is the currently favored route according to guidelines. Iron is administered according to the iron deficit calculated using Ganzoni's formula, i.e., iron deficit in $\mathrm{mg}=$ body weight $(\mathrm{kg}) \times[15-$ actual $\mathrm{Hb}$ in $\mathrm{g} / \mathrm{dL}] \times 2.4+500$. Usually, the required dose is around $1000 \mathrm{mg}$.

- Oral iron is not recommended because of poor tolerability, impaired gastrointestinal (Gl) absorption, drug interactions, and increased hepcidin levels precluding iron resorption from the gut.

- European Society of Cardiology (ESC) recommends treatment with IV ferric carboxymaltose in symptomatic heart failure patients with iron deficiency to improve heart failure symptoms and quality of life (Class Ila).

- American College of Cardiology/American Heart Association/ Heart Failure Society of America (ACC/AHA/HFSA) does not recommend any specific formulation but recommend IV iron in patients with heart failure and iron deficiency as a Class IIb recommendation. ${ }^{2}$

\section{Modification of Cardiac Medicines in the Presence of Anemia}

Iron supplements can interfere with the absorption of captopril and perhaps other angiotensin-converting-enzyme (ACE) inhibitors. Iron appears to bind with them resulting in a compound that the body cannot absorb. This, of course, also impairs iron absorption. To minimize this, iron supplements and ACE inhibitors should be taken at least 2-3 h apart. ACE inhibitors and angiotensin receptor blockers (ARBs) can also produce anemia by reducing erythropoietin. Erythropoietin should be avoided in heart failure and hypertension. For patients on antiplatelet therapy, one must meticulously look for Gl blood loss as the cause for anemia. If GI blood loss is present, the lowest dose of an appropriate antiplatelet regime should be prescribed.

\section{Kidney and Heart}

The heart and kidneys are inextricably linked in terms of hemodynamics and regulatory functions. The disease of one organ invariably affects the other. Heart failure and coronary artery disease (CAD) patients with underlying renal disease have a worse prognosis compared to patients with normal renal parameters.

\section{Heart Failure}

Renal dysfunction in heart failure is attributed to renal hypoperfusion, venous congestion, elevated intraabdominal pressure, increased renal interstitial pressure, and intrinsic renal disease. Even stable heart failure patients have some degree of renal impairment. About $50 \%$ of heart failure patients have chronic kidney disease (CKD) and $40 \%$ of CKD patients have heart failure. Cardiorenal syndrome (CRS) is a term used to define disorders of the heart and kidneys whereby "acute or chronic dysfunction in one organ may induce acute or chronic dysfunction of the other" (Table 2).

\section{Treatment of Heart Failure in Patients with Kidney Disease}

- ACE: They should be started at small doses with periodical titration based on potassium and creatinine levels. Because fluid retention attenuates the effect of ACE-I, they should be started after optimizing diuretics. ARBs can be prescribed to ACE-I intolerant cases (cough).

- Hydralazine + nitrates: it is used as an alternative in whom ACE-I/ ARBs cannot be given

- Diuretics: they remain the cornerstone of treatment in relieving symptoms. It is important to maintain potassium between 4 and $5 \mathrm{mmol} / \mathrm{L}$ to reduce the risk of hypokalemia precipitating arrhythmias or causing dangerous hyperkalemia. Torsemide has better oral bioavailability compared to furosemide and can be given once daily. Aldosterone inhibitors should be used cautiously when creatinine and potassium are elevated. Metolazone is a good "add-on" drug in patients with diuretic resistance.

\section{Coronary Artery Disease (CAD)}

CAD behaves differently in CKD. The unique features are early onset, rapid progression, increased vascular stiffness, more calcification, increased resistance to statins, and increased incidence of bleeding as well as higher rates of complications with percutaneous and surgical revascularization. In patients with CAD and CKD, it is advisable to use beta-blockers with predominant hepatic clearance, such as metoprolol and carvedilol and to avoid atenolol (Table 3).

\section{Treatment of Kidney Disease in the Presence of Cardiac Problem}

- Since many cardiac drugs increase serum potassium, careful monitoring of potassium by the physician or nephrologist is essential.

- Till further large-scale trial results are out, allopurinol may be preferred over febuxostat to treat hyperuricemia.

- Because of the hemodynamic instability in heart failure, coexisting end-stage renal disease (ESRD) patients may require a slower rate of fluid removal during dialysis.

Table 3: Uremic specific risk factors in cardiovascular disease (CVD)
1. Anemia
2. $\mathrm{PO}_{4}$ retention
3. Hyperparathyroidism
4. Vascular calcification
5. Hyperhomocystinuria
6. Hemosiderosis

Table 2: Types of cardiorenal syndrome

\begin{tabular}{ll}
\hline Type 1: acute cardiorenal syndrome & Acute heart failure leading to acute kidney failure \\
Type 2: chronic cardiorenal syndrome & Chronic heart failure leading to progressive, permanent damage to kidneys \\
Type 3: acute renocardiac & Acute kidney injury leading to acute heart disease \\
Type 4: chronic renocardiac & Chronic kidney disease leading to reduced cardiac function \\
$\begin{array}{l}\text { Type 5: secondary cardiorenal } \\
\text { a syndrome due to systemic disease }\end{array}$ & $\begin{array}{l}\text { Systemic diseases causing both cardiac and renal dysfunction, e.g., sepsis, } \\
\text { diabetes }\end{array}$ \\
\hline
\end{tabular}


- Contrast-induced nephropathy during coronary angiogram is an insult to the kidney in patients with CAD. It can be avoided by using the right contrast, correct dose as well as good hydration. The maximal allowable contrast dose (MACD) was first conceptualized by Cigarroa et al. and defined by the following empiric formula: MACD $=5 \mathrm{~mL}$ x body weight (in $\mathrm{kg}$ )/serum creatinine (in $\mathrm{mg} / \mathrm{dL}$ ), with a maximum dose of $300 \mathrm{~mL}^{3}$

\section{Obstructive Sleep Apnea}

It is an emerging risk factor for many cardiac conditions. Risk increases with the severity of obstructive sleep apnea (OSA) which is assessed by the apnea-hypopnea index (AHI). AHI is calculated by dividing the number of apnea/hypopnea events by the number of hours of sleep (Flowchart 1).

$\mathrm{AHI}<5$-normal

AHI 5-15-mild sleep apnea

AHI 15-30-moderate sleep apnea

$\mathrm{AHI}>30$ — severe sleep apnea

\section{Treatment of Obstructive Sleep Apnea}

Continuous positive airway pressure (CPAP) for at least $4 \mathrm{~h}$ combined with routine pharmacotherapy has been shown to reduce systemic blood pressure, improve heart failure symptoms and reduce the recurrence of arrhythmias. Patients should also be counseled on weight reduction, reduction in alcohol, and refraining from sedatives. Bariatric surgery can have a significant cardiac impact.

\section{Modification of Cardiac Medicines in Obstructive Sleep Apnea}

Obstructive sleep apnea should be suspected and ruled out using polysomnography in patients with refractory hypertension, unexplained heart failure, or atrial fibrillation.

It is better to avoid beta-blockers in these candidates as they can further increase the weight and aggravate OSA. The preferred antihypertensives will be ACE-I and ARBs. Aldosterone antagonists are the preferred diuretic agent if required.
Table 4: Pathogenic mechanism of chronic obstructive pulmonary disease and heart diseases

\begin{tabular}{ll}
\hline Traditional or shared risk factors & Overlapping pathways \\
\hline Cigarette smoking & Systemic inflammation \\
Aging and senescence & Oxidative stress \\
Environmental pollutants & Prothrombotic state \\
Sex & Activated renin-angiotensin \\
& system \\
Diet & Genetics, sedentarism \\
\hline
\end{tabular}

\section{Chronic Obstructive Pulmonary Disease}

Chronic obstructive pulmonary disease patients have an increased prevalence of heart failure and a 2-5-fold increased risk of CAD. Almost half of COPD patients die due to cardiovascular disease. COPD and heart diseases share many common risk factors and pathogenic mechanisms (Table 4).

\section{Treatment of Cardiac Problems in COPD Patients Coronary Artery Disease:}

Aspirin is proven to be safe and has been found to reduce COPD exacerbations. It should be the first-line antiplatelet agent. Cardioselective beta-blockers, such as bisoprolol are found to be effective in COPD patients in reducing the intensity and frequency of angina without a significant increase in airway resistance.

\section{Heart Failure:}

The use of cardioselective beta-blockers (e.g., bisoprolol) have been shown to reduce mortality even in the presence of COPD without significant reduction in FEV1. Ivabradine is a good alternative in patients who cannot tolerate beta-blockers to reduce heart rate. Digoxin may have increased susceptibility to develop toxicity in COPD and should be used with caution. Excessive diuresis may aggravate asthma due to dehydration.

Flowchart 1: Pathophysiological link between obstructive sleep apnea (OSA) and cardiovascular (CV) disease ${ }^{4}$

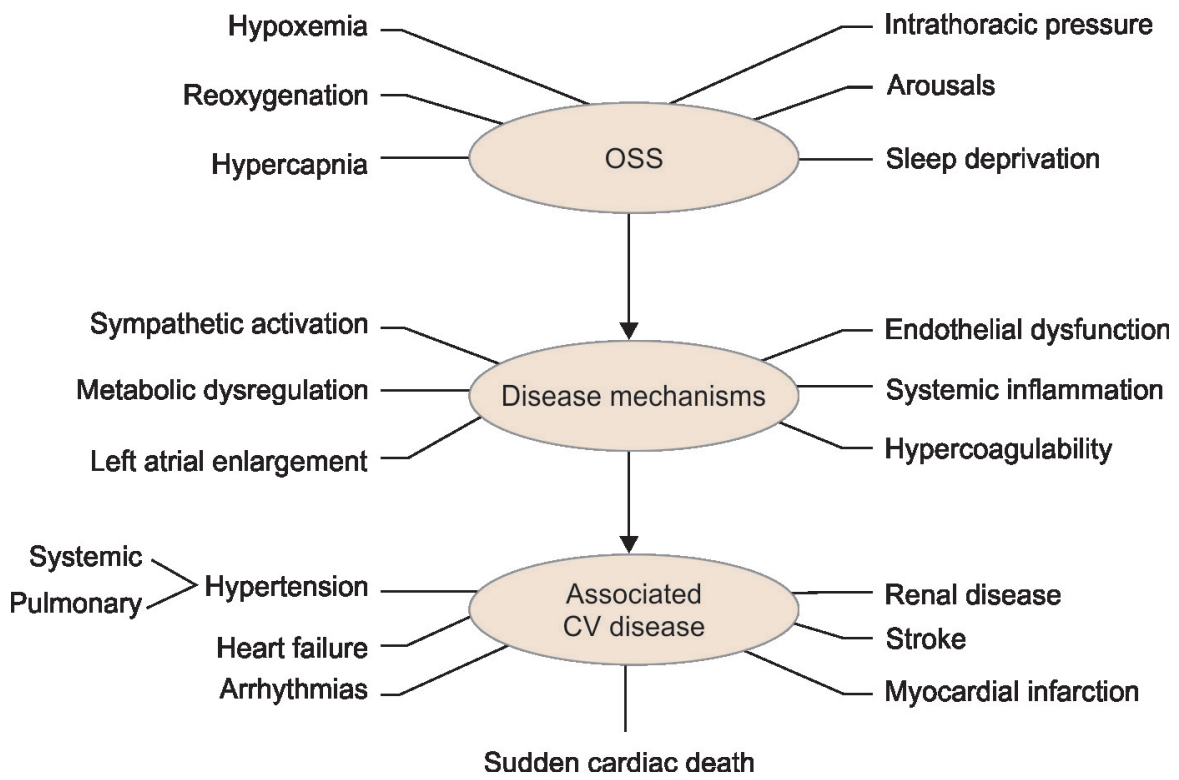




\section{Arrhythmias:}

Supraventricular and ventricular arrhythmias are more common compared to matched cohorts. This is to hypoxia, hypercapnia, and frequent electrolyte abnormalities. Multifocal atrial tachycardia is almost exclusively seen in COPD patients. Heart rate-limiting calcium channel blockers, such as verapamil, diltiazem may be used if left ventricle (LV) function is normal. Amiodarone should be reserved for severe arrhythmias as it tends to produce lung fibrosis.

\section{Management of COPD in Cardiac Patients}

Beta agonist bronchodilators are associated with a 1.5-4.5-fold increased risk of arrhythmias. The anticholinergic agent tiotropium was recently found to have better efficacy in at least moderate COPD and has demonstrated neutral safety outcomes. Theophylline and short-acting beta-agonist inhalers should be minimized because they increase the incidence of atrial fibrillation. During exacerbation episodes secondary to infections, azithromycin has to be avoided or used with caution after the estimation and monitoring of QTC interval.

\section{Diabetes and Heart}

Risk of cardiovascular events in diabetes

- $65 \%$ of diabetes deaths are due to cardiovascular disease.

- Coronary heart disease deaths: increased by 2-4-fold.

- Heart failure: increased by $2-5$-fold.

- Stroke risk: increased by 2-4-fold.

Diabetes can affect the heart in three ways (Flowchart 2):

1. Muscle

2. Arteries

3. Nerves

\section{Treatment of Cardiac Problems in Diabetic Patients}

\section{Coronary Artery Disease:}

In many, CAD remains silent without any symptoms; so apart from a routine stress test, patients should be screened for markers of subclinical atherosclerosis (microalbuminuria, ankle-brachial index, multislice CT angiogram). Microvascular angina is much more common than non-diabetics-hence individuals with angina despite normal epicardial coronaries by angiogram must be treated with nicorandil. Since diabetic subsets have increased thrombus load during ACS, prasugrel should be used in percutaneous coronary interventions $(\mathrm{PCl})$ cases whenever appropriate. All diabetics between the ages of 40 and 75 should receive statins if low-density lipoproteins (LDL) $>70 \mathrm{mg} / \mathrm{dL}$. Though evidence for the use of aspirin in primary prevention is weak, it can still be given to diabetic males $>5$ years and females $>65$ years with one additional risk factor (ADA guidelines). ${ }^{5}$

\section{Heart Failure:}

The initial abnormality is in the diastolic function of the heart due to impaired relaxation because of myocardial fibrosis. The tissue Doppler parameter of $E / E^{\prime}$ more than 15 predicts future heart failure in asymptomatic diabetics. Routine echocardiography is essential in all type II diabetic patients to pick up early heart muscle disease. Among beta-blockers, Carvedilol may offer an advantage in diabetics because of its favorable effect on insulin sensitivity and lipid profile. Angiotensin receptor-neprilysin inhibitors (ARNI) (sacubitril-valsartan) should be used instead of ACE-I and ARBs.

\section{Neuropathy:}

In the early stages, parasympathetic withdrawal can lead to unopposed sympathetic activity leading to increased heart rate and blood pressure resulting in increased oxygen demand. It can also lead to ventricular arrhythmias and sudden death. Later, there is sympathetic withdrawal also leading to postural hypotension causing a decrease in coronary hypoperfusion. This is also the same reason for silent deaths.

\section{Treatment of Diabetes in Cardiac Patients}

Drugs with a propensity to precipitate hypoglycemia like sulfonylureas and exogenous insulin should be used with caution. Metformin has remained the first-line therapy but consideration must be given in starting sodium-glucose cotransporter-2 (SGLT-2) inhibitors early in the treatment of diabetes because of its favorable cardiovascular effects. Dipeptidyl peptidase 4 (DPP-4) inhibitors must be avoided in patients with left ventricular dysfunction.

There are two aspects of the treatment of diabetes in cardiac patients:

1. To use drugs with cardiovascular safety and benefit profile.

2. To avoid hypoglycemia.

SGLT-2 inhibitors are safe and beneficial to the heart Metformin and DPP-4 inhibitors, such as sitagliptin are safe. In patients with LV dysfunction and heart failure, Pioglitazone must be avoided. Insulin and sulfonylureas are notorious to produce hypoglycemia.

Flowchart 2: Pathophysiology in diabetes and heart

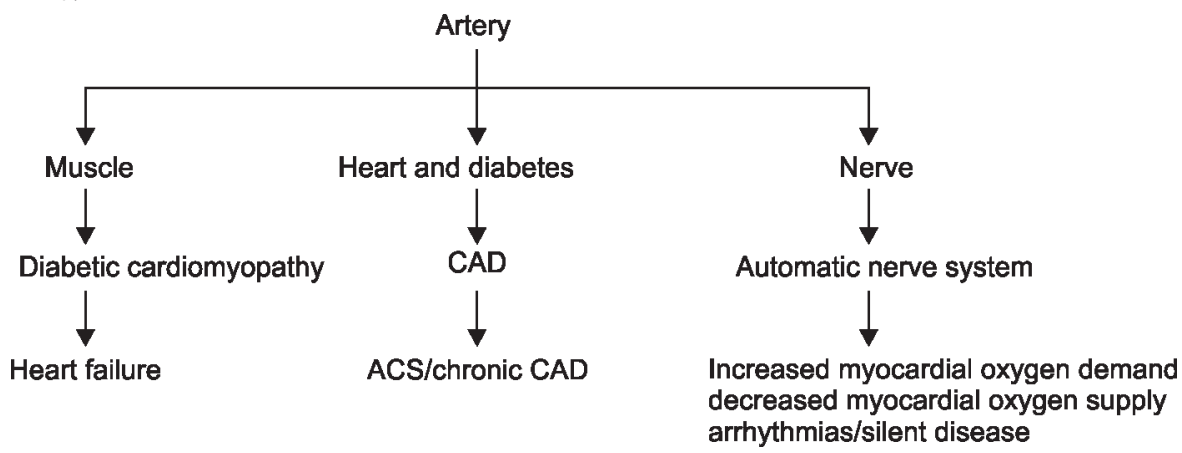


Optimizing Noncardiac Prescription in a Cardiac Patient

\begin{tabular}{|c|c|c|c|}
\hline Parameter & Normal & Hyperthyroid & Hypothyroid \\
\hline Heart rate (beats/min) & $72-84$ & $\begin{array}{l}\text { 88-130 } \\
\text { (palpitations) }\end{array}$ & $\begin{array}{l}\text { 60-80 } \\
\text { (fatigability, } \\
\text { dyspnea) }\end{array}$ \\
\hline Cardiac output (L/min) & $5-8$ & $>7$ & $<4.5$ \\
\hline Blood volume (\% of normal) & 100 & 105.5 & 84.5 \\
\hline $\begin{array}{l}\text { Systemic vascular } \\
\text { resistance }\end{array}$ & Normal & Reduced & Increased \\
\hline
\end{tabular}

\section{Thyroid and Heart}

Since thyroid hormones have a profound effect on the cardiovascular system, some of the symptoms of thyroid disorder may be due to cardiac and vascular abnormalities (Table 5).

\section{Hypothyroidism}

Common signs and symptoms include breathlessness, bradycardia, mild diastolic hypertension, narrowed pulse pressure. Progressive decline in cardiac contractility, diastolic dysfunction (earliest echo sign) pericardial effusion, and accelerated atherosclerosis are all observed.

\section{Hyperthyroidism}

Sinus tachycardia is the commonest rhythm disturbance, but atrial fibrillation causes the maximum concern. It occurs in $2-20 \%$ of hyperthyroid patients. Abnormal resting heart rate, irregular heart rate, and tremors can often give a clue at the bedside.

\section{Treatment of Thyroid Disorders in Cardiac Patients Hypothyroidism}

In patients with heart disease and the elderly, thyroxine must be started at low doses (25-50 $\mu \mathrm{g} /$ day) and increased gradually every 6-8 weeks. Heart rate-limiting drugs should be used with caution. Dihydropyridine calcium antagonists, such as amlodipine can aggravate pedal edema.

\section{Hyperthyroidism}

Beta-blockers are the best drugs to control heart rate along with antithyroid drugs.

\section{Treatment of Cardiac Problem in Patients with Thyroid Disorders}

Prolonged untreated thyroid disorder (hypo or hyper) can lead to cardiac failure similar to dilated cardiomyopathy, which is usually correctable after treating the basic thyroid problem. In the treatment of arrhythmias, amiodarone may pose a problem as it can produce both hypo and hyperthyroidism, so frequent monitoring of thyroid function is necessary. Anticoagulation treatment in
Table 6: Diagnostic criteria to diagnose obesity and central obesity in India

\begin{tabular}{ll}
\hline Obesity in India & Normal: $18-22.9 \mathrm{~kg} / \mathrm{m}^{2}$ \\
& Overweight: $23-24.9 \mathrm{~kg} / \mathrm{m}^{2}$ \\
& Obesity: $>25 \mathrm{~kg} / \mathrm{m}^{2}$ \\
Central obesity & Waist circumference \\
& Males $>89 \mathrm{~cm}$, females $>77 \mathrm{~cm}$ \\
\hline
\end{tabular}

atrial fibrillation needs meticulous monitoring for international normalized ratio (INR) if patients are on vitamin $\mathrm{K}$ antagonists.

\section{Obesity and Heart}

Obesity is classified according to body mass index (BMI) or waist circumference (central obesity) (Table 6).

\section{Treatment of Obesity in Cardiac Condition (Indian (riteria) ${ }^{6}$}

- Diet, lifestyle modification.

- Pharmacotherapy: antiobesity drugs without cardiac side effects (preferable orlistat) is indicated in patients with BMI $>27 \mathrm{~kg} /$ $\mathrm{m}^{2}$ or $>25 \mathrm{~kg} / \mathrm{m}^{2}$ if associated with at least 1 obesity-linked comorbidity.

- Bariatric surgery: indicated if BMI $>37.5 \mathrm{~kg} / \mathrm{m}^{2}$ or $>32.5 \mathrm{~kg} / \mathrm{m}^{2}$ if associated with comorbidities like sleep apnea, hypertension, or diabetes.

\section{Treatment of Cardiac Conditions in Obesity}

- Beta-blockers can be avoided; if necessary, bisoprolol or carvedilol can be used.

- Avoid drugs that can increase weight. ACE-I and ARBs are probably safe.

\section{Chemotherapy and Heart}

Due to the increasing prevalence of cancer and the wide therapeutic options that are available today, basic knowledge about chemotherapy and heart is essential. It is important to predict and prevent cardiotoxicity due to chemotherapy in cancer patients (Table 7).

\section{Use of Chemotherapy to Prevent Cardiac Toxicity \\ Evaluation of Cardiac Toxicity:}

LV ejection fraction is one of the most important predictors of adverse outcomes. It is best assessed by the echo. Newer modalities in echo-like speckle tracking and strain rate imaging can predict outcomes much better than ejection fraction (EF) by the 2D echo. The global longitudinal strain (GLPS) is emerging as the most

Table 7: Risk factors for cancer treatment-induced cardiotoxicity

\begin{tabular}{ll}
\hline Existing comorbidities & Patients with diabetes, kidney disease \\
Prior cardiovascular disease & Reduced LV function, CAD, prior arrhythmias \\
$\begin{array}{l}\text { Presence of risk factors for sudden cardiac death } \\
\text { Chemotherapeutic agent }\end{array}$ & QT prolongation, electrolyte abnormalities, proarrhythmic drugs \\
& $\begin{array}{l}\text { High-dose anthracycline therapy, low-dose anthracycline or } \\
\text { trastuzumab therapy in high-risk cardiac patients }\end{array}$ \\
Radiotoxicity/combined treatment & $\begin{array}{l}\text { High-dose cardiac radiation therapy, inability of cardiac avoidance (anterior } \\
\text { or left chest radiation), combined chemo- and radiotherapy }\end{array}$ \\
\hline
\end{tabular}




\begin{tabular}{ll} 
Table 8: Preventive strategies for cancer therapeutics induced cardiotoxicity \\
\hline Before cardiotoxic cancer therapy & $\begin{array}{l}\text { Control of modifiable risk factors } \\
\text { Optimize cardiovascular medications }\end{array}$ \\
During cardiotoxic cancer therapy & $\begin{array}{l}\text { Prioritize liposomal formulation and continuous infusion of doxorubicin } \\
\text { Use of lowest effective radiation dose } \\
\text { Discontinue chemo- and radiotherapy when appropriate } \\
\text { Frequent cardiac evaluation, echo for appropriate patients }\end{array}$ \\
After cardiotoxic cancer therapy & $\begin{array}{l}\text { Monitor cardiovascular health, cardiac function } \\
\text { Control modifiable risk factors }\end{array}$ \\
\hline
\end{tabular}

robust technique to predict and prognosticate early cardiotoxicity. Echocardiography has to be done before, during, and after cancer therapy (chemotherapy and radiotherapy) (Table 8).

\section{Use of Cardiac Drugs in Cancer Patients}

Valsartan is an important drug to prevent and treat cardiac toxicity. The patient has to be followed up for at least 5 years to detect cardiac diseases.

\section{Gastrointestinal System and Heart}

Patients with cardiac problems may be prone to GI diseases due to heart failure itself or due to drugs like aspirin, dual antiplatelet drugs, or anticoagulants.

\section{Treatment of Cardiac Conditions in the Presence of GI Problems}

- Gl bleed: It is important to use the correct combination and dose of antiplatelets. Aspirin must be used only in select highrisk patients for primary prevention (increased CV risk and no risk of bleeding). In secondary prevention, low-dose aspirin (100 mg) must be used during dual antiplatelet therapy. Dual antiplatelet therapy (DAPT) must be continued after 1 year only in select patients (ACS, post $\mathrm{PCl}$ post-CABG). Antiplatelets must be withheld during the acute stage and restarted only when there is no active bleeding. On restarting, low-dose aspirin along with proton-pump inhibitors (PPI) has been shown to reduce the risk of re-bleed.

- Liver failure: Since many cardiac drugs are metabolized in the liver, caution should be taken while prescribing them (e.g., amiodarone, warfarin, labetalol). Statins are safe. Statins must be stopped only when liver enzymes increase more than three times the upper limit of normal. Since the risk of bleeding is high, patients on blood thinners should be carefully monitored and should be put on the lowest effective dose.

\section{Use of GI Drugs in Cardiac Patients}

- Use of QT-prolonging drugs like cisapride, domperidone, certain PPIs (by causing hypomagnesemia) should be used with caution.

- Since certain studies have shown an interaction between omeprazole and clopidogrel, it is better to use other PPIs when the patient is on clopidogrel. Use of PPI in CAD. ${ }^{7}$

- Class I indication: history of prior gastrointestinal bleeding.

- Class Ila: In patients with increased risk of gastrointestinal bleeding, including thosewith advanced age and those with concomitant use of warfarin, steroids, or nonsteroidal antiinflammatory drugs.
- Class III: Routine use of PPIs is not recommended for patients at low risk of $\mathrm{Gl}$ bleeding.

\section{Psychiatry and Heart}

Commonly encountered psychosocial and behavioral disorders include:

- Depression: It is three times more common in cardiac patients compared to controls. A total of $15-30 \%$ of cardiac patients have depression. It also aggravates the basic cardiovascular disease directly and indirectly through lifestyle changes.

- Anxiety: It increases cardiac vascular events due to increased cardiac output and arrhythmias. A combination of anxiety and depression is more dangerous than either of them alone.

- Anger and hostility: Studies have shown that anger and hostility are associated with a 19\% greater risk of developing cardiovascular disease.

- Stress: INTERHEART study showed that patients experiencing first myocardial infarctions (MI) had greater work stress, stress at home, general stress, and permanent stress after correcting for the demographic area and medical factors. ${ }^{8}$

\section{Treatment of Psychiatric Problem in Cardiac Patients}

Short-term use of selective serotonin reuptake inhibitors (SSRI) reduces depressive symptoms as well as reduces re-hospitalization. Potential side effects include sexual dysfunction and increased bleeding tendency (with fluoxetine). Tricyclic antidepressants (TCAs) may not be safe because of weight gain, fluid overload, and prolonged QT interval. Benzodiazepines are the preferred agents in treating anxiety. Their use is conceived as a short-term and intermittent treatment program in acute coronary syndrome, hypertension, and heart failure.

\section{Modification of Cardiac Drugs in Psychiatric Patients}

As far as cardiac drugs are concerned, older beta-blockers should be avoided.

Amiodarone should not be combined with TCAs.

\section{Autoimmune Diseases and Heart}

This includes systemic lupus erythematosus (SLE), rheumatoid arthritis, ankylosing spondylitis, and scleroderma. Patients with autoimmune disorders have an increased risk of CVD morbidity and premature mortality. This is to a major extent due to the chronic inflammatory burden and to reduced physical activity. Lessons from rheumatoid arthritis (RA) and SLE may have implications for future research on the pathogenesis of atherosclerotic vascular disease in general. Recent data indicate that suppression of inflammation reduces the risk of CVD morbidity and mortality in patients with severe RA. 


\section{Treatment of Autoimmune Disorder in Cardiac Patients}

Data suggest that no nonsteroidal antiinflammatory drugs (NSAID) or cyclooxygenase-2 (Cox 2) inhibitor is entirely safe and naproxen has a reasonably better cardiovascular profile due to its antiplatelet effect. ${ }^{9}$ Because of the high incidence of Gl side effects, most guidelines recommend the addition of a PPI to an NSAID.

\section{Cardiac Prescription in Autoimmune Disorders}

The antihypertensive that will work despite NSAIDs is dihydropyridine (DHP) calcium antagonists. ACE-I, ARBs, and beta-blockers may not be effective with NSAIDs.

\section{Conclusion}

More than two-thirds of cardiac patients have at least one of the above-mentioned comorbid conditions. So, one should have thorough and updated knowledge to ensure that both the cardiac problem and the coexisting comorbid condition are treated in the best possible way to reduce the impact of noncardiac drugs on the cardiac system and vice versa. It is also preferable to use drugs that will benefit both cardiac and noncardiac conditions. This will ensure maximum efficacy and safety of treatment for the underlying disease conditions.

\section{References}

1. Dunlay SM, Westo SA, Redfield MM, et al. Anemia and heart failure: a community study. Am J Med 2008;121(8):726-732. DOI: 10.1016/j. amjmed.2008.03.039.

2. Yancy CW, Jessup M, Bozkurt B, et al. 2017 ACC/AHA/HFSA focused update on the guidelines for the management of heart failure: a report of the American college of cardiology/American Heart Association task force on clinical practice. Circulation 2017;136:e137e161. DOI: 10.1161/CIR.0000000000000509.
3. Cigarroa RG, Lange RA, Williams RH, et al. Dosing of contrast material to prevent contrast nephropathy in patients with renal disease. Am J Med 1989;86(6 Pt 1):649-652. DOI: 10.1016/0002-9343(89) 90437-3.

4. Virend K Somers, David P White, Raouf Amin, et al. Sleep apnea and cardiovascular disease: an American Heart Association/American College of Cardiology Foundation Scientific Statement from the American Heart Association Council for High Blood Pressure Research Professional Education Committee, Council on Clinical Cardiology, Stroke Council, and Council on Cardiovascular Nursing. J Am Coll Cardiol 2008;52(8):686-717. DOI: 10.1016/j.jacc.2008.05.002.

5. American Diabetes Association. 10. Cardiovascular disease and risk management: standards of medical care in diabetes-2019. Diab Care 2019;42(Supplement 1):S103-S123. DOI: 10.2337/dc19-S010.

6. BehI S, Misra A. Management of obesity in adult Asian Indians. Indian Heart J 2017;69(4):539-544. DOI: 10.1016/j.ihj.2017.04.015.

7. Levine GN, Bates ER, Bittl JA, et al. 2016 ACC/AHA guideline focused update on duration of dual antiplatelet therapy in patients with coronary artery disease: a report of the American College of Cardiology/American Heart Association Task Force on Clinical Practice Guidelines: an update of the 2011 ACCF/AHA/SCAI guideline for percutaneous coronary intervention, 2011 ACCF/AHA guideline for coronary artery bypass graft surgery, 2012 ACC/AHA/ACP/AATS/ PCNA/SCAI/STS guideline for the diagnosis and management of patients with stable ischemic heart disease, 2013 ACCF/AHA guideline for the management of ST-elevation myocardial infarction, 2014 AHA/ACC guideline for the management of patients with non-STelevation acute coronary syndromes, and 2014 ACC/AHA guideline on perioperative cardiovascular evaluation and management of patients undergoing noncardiac surgery. Circulation 2016;134(10):e123-e155. DOI: $10.1161 / C I R .0000000000000404$.

8. Yusuf S, Hawken S, Ounpuu S, et al. Effect of potentially modifiable risk factors associated with myocardial infarction in 52 countries (the INTERHEART study): case-control study. Lancet 2004;364(9438):937952. DOI: 10.1016/S0140-6736(04)17018-9.

9. Pawlosky N. Cardiovascular risk: are all NSAIDs alike? Can Pharm J (Ott) 2013;146(2):80-83. DOI: 10.1177/1715163513481569. 